\title{
Approaching the Symbol Grounding Problem with Probabilistic Graphical Models
}

\author{
Stefanie Tellex, ${ }^{*}$ Thomas Kollar, ${ }^{*}$ \\ Steven Dickerson, Matthew R. Walter, Ashis Gopal Banerjee, Seth Teller, Nicholas Roy
}

\begin{abstract}
- In order for robots to engage in dialogue with human teammates, they must have the ability to identify correspondences between elements of language and aspects of the external world. A solution to this symbol-grounding problem (Harnad, 1990) would enable a robot to interpret commands such as "Drive over to receiving and pick up the tire pallet." This article describes several of our results that use probabilistic inference to address the symbol-grounding problem. Our approach is to develop models that factor according to the linguistic structure of a command. We first describe an early result, a generative model that factors according to the sequential structure of language, and then discuss our new framework, generalized grounding graphs $\left(G^{3}\right)$. The $G^{3}$ framework dynamically instantiates a probabilistic graphical model for a natural language input, enabling a mapping between words in language and concrete objects, places, paths, and events in the external world. We report on corpus-based experiments in which the robot is able to learn and use word meanings in three real-world tasks: indoor navigation, spatial language video retrieval, and mobile manipulation.
\end{abstract}

A $s$ robots move out of the lab and into the real world, it is critical to develop ways for human users to easily and flexibly command them. Natural language dialogue is a compelling solution to this problem because the operator can flexibly express complex requirements, enabling interaction with the robot as if it were another human. In order to engage effectively in dialogue, a robot must be able to interpret natural language commands. For example, a human supervisor might tell an autonomous forklift, "Put the tire pallet on the truck" (figure 1a), or an operator might command a humanoid robot, "Drive down the hall past the elevators" (figure 1b.)

A critical component to understanding commands like these is the ability to map words in the language to aspects of the external world. This mapping, which Harnad (1990) called the symbol-grounding problem, has been studied since the early days of artificial intelligence. There are broadly three different ways people have approached the symbol-grounding problem in robotics. Starting with Winograd (1970), many have manually created symbol systems that map between language and the external world, connecting each term onto a prespecified action space and set of environmental features (Bugmann et al. 2004; Dzifcak et al. 2009; Hsiao, Mavridis, and Roy 2003; Kress-Gazit and Fainekos 2008; MacMahon, Stankiewicz, and Kuipers 2006; Roy, Hsiao, and Mavridis 2003; Roy 2005). This class of systems takes advantage of the structure of spatial language, but usually the systems do not involve learning, have little perceptual feedback, and have a fixed action space. A second approach involves learning the meaning of words in the sensorimotor space (for example, joint angles and images) of the robot (Marocco et al. 2010; Modayil and Kuipers 2007; Sugita and Tani 2005). By

*The first two authors contributed

equally to this article. 


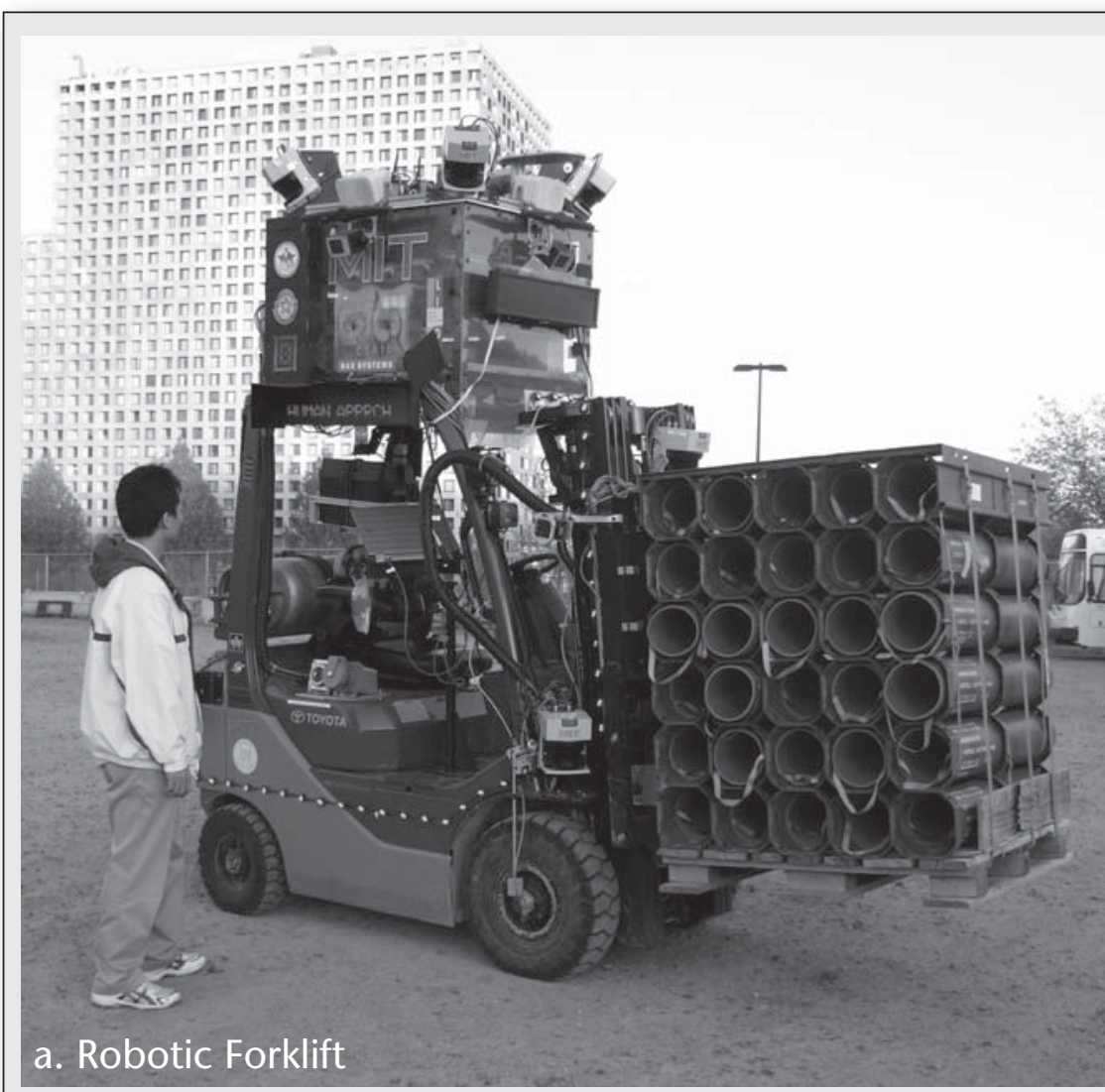

Pick up the pallet of boxes in the middle and place them on the trailer to the left.

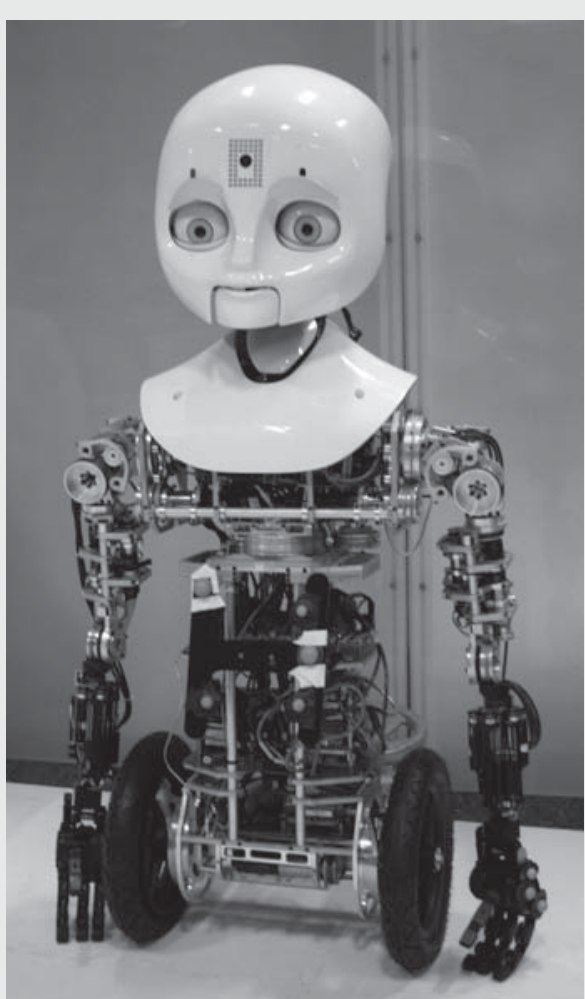

b. Humanoid Robot

Go down the hall past the elevators to the kitchen.

Figure 1. Robotic Platforms and Commands.

treating linguistic terms as a sensory input, these systems must learn directly from complex features extracted by perceptual systems, resulting in a limited set of commands that they can robustly understand. A third approach is to use learning to convert from language to aspects of the environment. These approaches may use only linguistic features (Ge and Mooney 2005; Shimizu and Haas 2009), spatial features (Regier 1992) or linguistic, spatial, and semantic features (Branavan et al. 2009; Branavan, Silver, and Barzilay 2011; Kollar et al. 2010b; Matuszek, Fox, and Koscher 2010; Vogel and Jurafsky 2010). These approaches learn the meaning of spatial prepositions (for example, "above" [Regier 1992]), verbs of manipulation (for example, "push" and "shove" [Bailey 1997]), and verbs of motion (for example, "follow" and "meet" [Kollar et al. 2010a]) and landmarks (for example, "the doors" [Kollar et al. 2010b]).

In this article, we give an overview of our probabilistic approach to the symbol-grounding problem. By taking a probabilistic approach, we are able to build systems that learn word meanings from large corpora of examples and use those meanings to find good groundings in the external world, despite uncertainty. Our first approach uses a generative model that factors according to the sequential structure of language. This model can be used to follow natural language route instructions and to perform spatial language video retrieval. However, the generative approach requires explicit corpora for each modeled factor, rather than learning word meanings directly from in-domain language. It cannot represent complex linguistic structures such as referring expressions (for example, "the door across from the elevators") and multiargument verbs (for example, "put the pallet on the truck"). To address these limitations, we developed a new framework, called generalized grounding graphs $\left(\mathrm{G}^{3}\right)$, introduced in Tellex et al. (2011). The $\mathrm{G}^{3}$ framework dynamically instantiates a conditional probabilistic graphical model that factors according to the compositional and hierarchical structure of a natural language phrase. Using the new model, we created a system that successfully follows many mobile-manipulation commands from a corpus created by untrained annotators using crowd sourcing.

Several earlier publications describe the primary technical contributions of the models (Kollar et al. 
2010a, 2010b; Tellex et al. 2010, 2011). This article provides an integrated overview of results and lessons learned for the generative model and the $\mathrm{G}^{3}$ framework in three domains: navigation in indoor environments, spatial language video retrieval, and mobile manipulation.

\section{Approach}

Our goal is a framework that can map between language and the external world. We assume a natural language utterance $\Lambda$ has a corresponding set of groundings in the external world. Groundings can be objects (for example, a truck or a door), places (for example, a particular location in the world), paths (for example, a trajectory through the environment), and events (for example, a sequence of robot actions). We additionally assume a semantic map $m$, consisting of the locations and labels of other objects in the environment. The distribution we want to model is:

$$
p(\Lambda, \Gamma, m)
$$

We explicitly represent the joint distribution rather than the conditional because it can be used to solve several types of problems. To interpret commands, one can optimize over candidate groundings; this article describes our work in this area. Furthermore, the model could be used to generate natural language descriptions of objects or events by searching for a description $\Lambda$. Finally it could also be used to recognize events by directly computing the probability of a particular set of values and thresholding. The challenge in making this approach practical for any of these problems is factoring the distribution and providing models for each factor.

\section{Generative Model}

In our previous work (Kollar et al. 2010b), we approached this problem by factoring equation 1 to give $p(\Lambda \mid \Gamma, m) \times p(\Gamma, m)$. This formulation allowed us to make independence assumptions corresponding to the sequential clause structure of the language, yielding:

$$
p(\Lambda \mid \Gamma, m)=\prod_{i} p\left(\lambda_{i} \mid \Gamma, m\right)
$$

where $\lambda_{i}$ are the words associated with each clause. We assumed that each factor had a fixed structure: a verb, $v$, a spatial relation, $s r$, and a landmark, $l$. Furthermore, we assumed that each clause had a fixed set of groundings, consisting of a path fragment, $p$, and an object, $o$ :

$$
p\left(\lambda_{i} \mid \Gamma, m\right)=p(v, s r, l \mid p, o, m)
$$

We then made independence assumptions based on this structure.

$$
\begin{aligned}
& p(v, s r, l \mid p, o, m) \\
& =p(v \mid p) \times p(s r \mid p, o) \times p(l \mid p, m)
\end{aligned}
$$

This approach allowed us to define individual models for each term in the factorization. We defined models for prespecified verbs and adverbs such as "left," "right," and "straight," and we trained models for spatial relations such as "to," "past," and "through." For the landmark factor, we exploited co-occurrence statistics from a large online database ${ }^{1}$ of labeled images to estimate the probability of an unknown landmark phrase given objects detected in the semantic map (Kollar and Roy 2009). These statistics enabled the robot to estimate the probability of seeing a landmark phrase such as "the kitchen" using a limited set of existing object detectors, such as a refrigerator and a sink.

We tested our generative model in two realworld domains: following natural language directions through real-world environments and spatial language video retrieval. The model can be used to follow natural language directions by finding the path through the environment, $\gamma_{\text {path }} \in \Gamma$, that maximizes the distribution in equation 3 . To evaluate the system at following natural language directions, we collected from 15 subjects a corpus of 150 directions through a large office environment. Our system successfully followed 67 percent of the directions in the corpus, compared to human performance of 85 percent.

For video retrieval, the task was to find video clips from a large corpus (Roy et al. 2006) that match a spatial language description of a person's motion, such as "Show me people walking into the kitchen." The system performed ranked retrieval by scoring video clips according to how well they matched a spatial language query according to equation 3 . We demonstrated that our system could effectively retrieve video clips, evaluating on a large corpus of natural language queries created by untrained users. Figure 2 shows a sample query result for the system.

However, the generative framework has several limitations. First, because it only models the flat sequential structure of language, rather than the hierarchical structure, it cannot handle commands such as "Go to the door across from the elevators." The phrase "the door across from the elevators" is treated as a bag of words, and the system is unable to distinguish whether to approach the door or the elevators. Second, we assumed that each clause has a fixed structure consisting of a path and a landmark, but language has variable, hierarchical structure. The flat structure cannot support two-argument verbs like "Put the tire pallet on the truck," or nested arguments. Third, it is difficult to obtain models for the meanings of words in the individual factors. In our route directions data set, people 
often used "thru" instead of "through." Since the system learned word meanings from a separate, curated corpus that did not contain "thru," we manually encoded this synonymy. The system was unable to learn word meanings directly from the corpus.

\section{Generalized Grounding Graphs}

Our aim in creating the $\mathrm{G}^{3}$ framework was to address the challenges from the previous section by modeling the hierarchical, compositional structure of language in a framework that could learn word meanings from data. To facilitate learning, we converted the distribution in equation 1 to a discriminative model (Kollar, Tellex, and Roy 2010) by introducing a correspondence vector, $\Phi$ :

$$
p(\Phi \mid \Gamma, \Lambda, m)
$$

The correspondence vector $\Phi$ contains a Boolean variable $\phi$ for each linguistic constituent $\lambda \in \Lambda$ and corresponding grounding $\gamma \in \Gamma$ such that $\phi$ is true if $\lambda$ and $\gamma$ correspond and false otherwise.

The $\mathrm{G}^{3}$ framework factors the model according to the structure of the language, allowing explicit inference over groundings for each linguistic constituent:

$$
p(\Phi \mid \Lambda, \Gamma, m)=\prod_{i} p\left(\phi_{i} \mid \lambda_{i}, \Gamma, m\right)
$$

This factorization can be represented graphically as a factor graph (Kschischang, Frey, and Loeliger 2001). A factor graph is a bipartite graph with two types of nodes: random variables and factors. Each factor node corresponds to a factor in the distribution and connects to variable nodes, which are its arguments. For example, figure 3 shows a factor graph for the phrase "the truck," consisting of a single factor and three variables: $\gamma$, which is a vector of features corresponding to an object in the external world with a particular appearance and location, $\lambda$, the words "the truck," and $\phi$, which is true if $\gamma$ corresponds to $\lambda$, and false otherwise. The graph corresponds to the distribution $p(\phi \mid \gamma, \lambda, m)$. (If $\lambda$ in figure 3 were the words "the tire pallet," then $p(\phi=$ False $\mid \gamma, \lambda)$ would have higher probability.) Since the semantic map $m$ appears in all factors, we omit it from the graphical representation. We refer to factor graphs created by the $\mathrm{G}^{3}$ framework as grounding graphs. Word models in each factor can be learned discriminatively, and the resulting factorization allows the system to compose them in order to follow novel commands that may have never been seen in training.

In order to precisely define the factorization in equation 6, we use spatial description clauses (SDCs). SDCs were introduced by Kollar et al. (2010b) and refined by Tellex et al. (2011); they correspond to the parse structure of a natural language command. An SDC consists of a figure phrase $f$, a relation $r$, and a variable number of landmark

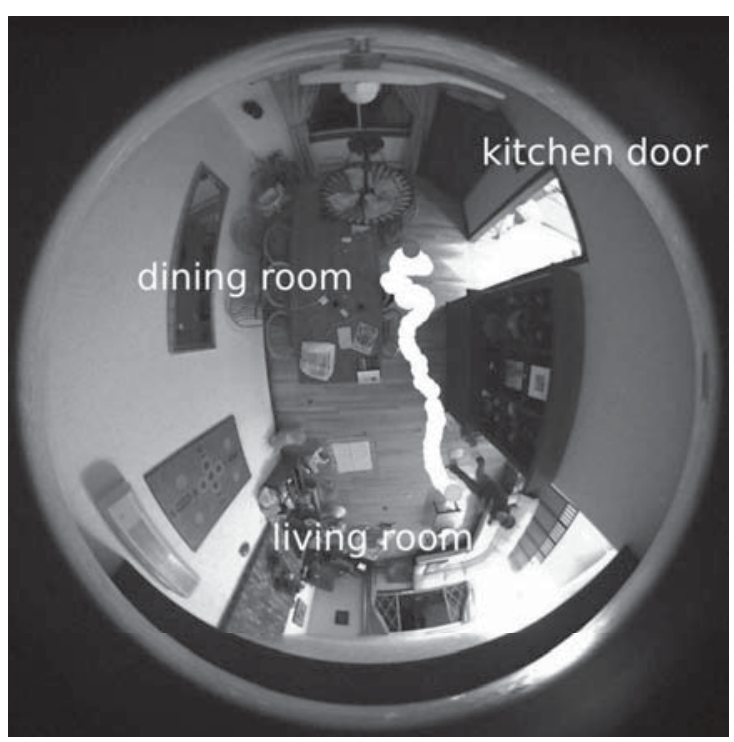

Highest-Ranked Clip
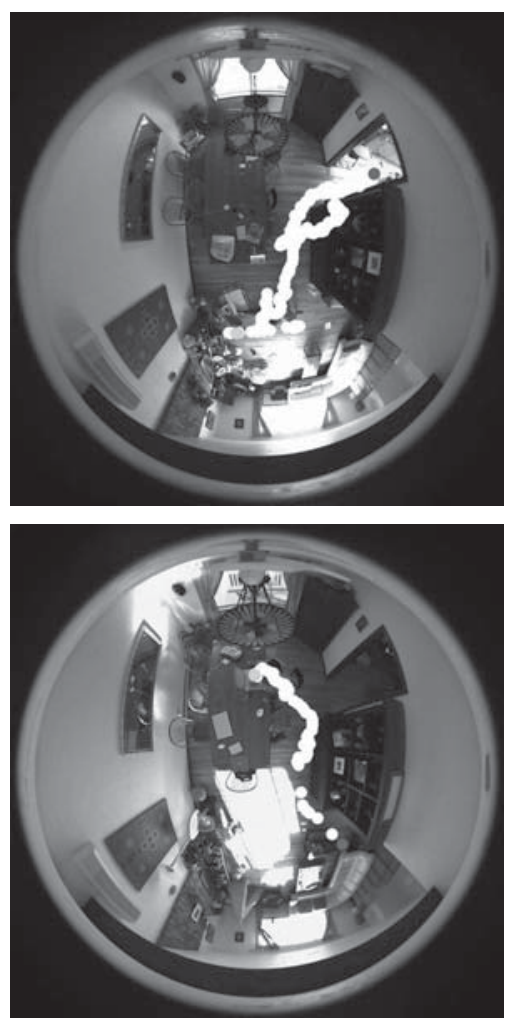

Low-Ranked Clips

Figure 2. Video Retrieval Results.

Query: "from the couches in the living room to the dining room table" (from Tellex et al. 2010). 
$O B J(f=$ the truck $)$

\section{a. SDC Tree}

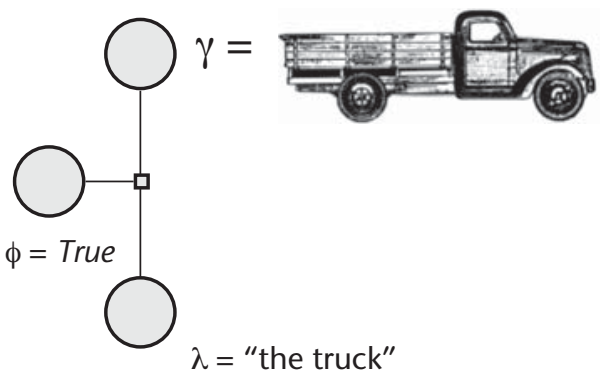

\section{b. Induced Model}

$p(\Phi \mid \Lambda, \Gamma, m)=p(\phi \mid \gamma, \lambda, m)$

c. Factorization

Figure 3. SDCs and Grounding Graph for the Phrase "the Truck."

noun phrases $l_{i}$. We assign a type to each SDC following the system defined by Jackendoff (1983):

EVENT Something that takes place (or should take place) in the world, for example, "Move the tire pallet" or "Turn right."

OBJECT A thing in the world. This category includes people and the robot as well as physical objects (for example, "forklift," "the tire pallet," "the hallway," "the person").

PLACE Places in the world (for example, "on the truck," "next to the tire pallet," or "in the kitchen").

PATH Paths through the world (for example, "past the truck" or "down the hall").

SDCs with relations contain one or more arguments. Since almost all relations take two core arguments or fewer, we use at most two landmark fields $l_{1}$ and $l_{2}$. Given this definition, a general natural language command is represented as a sequence of SDC trees. An SDC tree for the command "Put the pallet on the truck" appears in figure 5a. Leaf SDCs in the tree contain only text in the figure field, such as "the truck" (figure 3a). Internal SDCs contain text in the relation field and child SDCs in the figure and landmark fields.

The system automatically extracts SDCs from the Stanford dependency parse structure (de Marneffe, MacCartney, and Manning 2006). The SDC extraction algorithm maps between particular dependency types and fields in the SDCs, putting verbs and prepositions in the relation field, their arguments in the landmark field, and their subjects in the figure field. In cases of ambiguity the algorithm outputs multiple candidate SDCs for a single parse. We obtain additional candidates by running the extractor on the $n$-best list of parse candidates. The system then performs discriminative reranking using a model trained from annotated SDCs.

Using SDCs, we can rewrite the inner term from equation 6 as:

$$
p\left(\phi_{i} \mid \lambda_{i}, \Gamma, m\right)=p\left(\phi_{i} \mid S D C_{i}, \Gamma, m\right)
$$

Further independence assumptions can be made in the product terms based on the structure of the language. To specify these factors, we first define the variables in the model as follows:

$\phi_{i}$ True if the grounding $\gamma_{i}$ corresponds to ith SDC.

$\lambda_{i}$ The text of the figure field of the ith SDC.

$\lambda_{i}^{r}$ The text of the relation field of the ith SDC.

$\gamma_{i}^{f}, \gamma_{i}^{l 1}, \gamma_{i}^{l 2} \in \Gamma$ The groundings associated with the corresponding field of the ith SDC: the robot or object state sequence, or a location in the semantic map.

Looking at equation 7, we can see that the model has a factor for each SDC in the parse. The dynamically generated factors fall into two types:

$p\left(\phi_{i} \mid \lambda_{i}^{f}, \gamma_{i}, m\right)$ for leaf SDCs.

$p\left(\phi_{i} \mid \lambda_{i}^{r}, \gamma_{i}^{f}, \gamma_{i}^{l 1}, m\right)$ or $p\left(\phi_{i} \mid \lambda_{i}^{r}, \gamma_{i}, \gamma_{i}^{l 1}, \gamma_{i}^{l 2}, m\right)$ for internal SDCs.

Leaf factors always correspond to an OBJECT or PLACE SDC and operate over the correspondence variable $\phi_{i}$, the figure text $\lambda_{i}^{f}$, and a unique grounding $\gamma_{i}$. An internal factor corresponds to an OBJECT, PLACE, PATH, or EVENT SDC, which has text in the relation field. The arguments to these factors are correspondence variable $\phi_{i}$, relation text $\lambda_{i}^{r}$, and the candidate groundings $\gamma_{i}^{f}$ and $\gamma_{i}^{l 1}$ (and optionally $\gamma_{i}^{l 2}$ ) corresponding to the figure and landmark fields of an SDC.

For example, figure 4 shows the grounding graph for the phrase "on the truck." It contains a subgraph corresponding to "the truck" that is identical to the one shown in figure 3 . The value of the correspondence variable $\phi_{1}$ depends only on the values of $\lambda_{1}$ ("on") and the groundings $\gamma_{1}$ (a place in the world) and $\gamma_{2}$ (an object), and not on the specific words "the truck." This independence assumption enables the model to represent a general meaning for "on" that does not depend on specific text in its argument phrase.

Each factor in equation 7 is a log-linear model with the following form (Lafferty, McCallum, and Pereira 2001):

$$
\begin{aligned}
& p\left(\phi_{i} \mid \mathrm{SDC}_{i}, \Gamma, m\right) \\
& =\frac{1}{Z} \exp \left(\sum_{k} \mu_{k} s_{k}\left(\phi_{i}, \mathrm{SDC}_{i}, \Gamma, m\right)\right)
\end{aligned}
$$

Here, $s_{k}$ are feature functions (described more fully in Tellex et al. [2011]) that take as input a correspondence variable, an SDC, and a set of ground- 
ings and output a binary value. For example, one of the many feature functions corresponds to whether the landmark grounding $\gamma_{i}^{l}$ is supporting the figure grounding $\gamma_{i}^{f}$ and the word "on" is in the relation field of the SDC:

$$
f\left(\gamma_{i}^{f}, \gamma_{i}^{l}, \lambda_{i}^{r}\right) \equiv \operatorname{supports}\left(\gamma_{i}^{f}, \gamma_{i}^{l}\right) \wedge\left(\text { "on" } \in \lambda_{i}^{r}\right)
$$

We use features relating the distance between the figure and the landmark groundings, as well as the change in state at the beginning and end of the robot's trajectory. Features are created based on the syntactic role of the words in the language: whether it appears as a figure, relation, or landmark in the SDC. To ground noun phrases, the system assumes access to an object detector that can recognize certain classes of objects, such as pallets and trucks. The system learns to map between these labels and words that actually appeared in the command, such as "skid" or "trailer." We also use features derived from co-occurrence statistics from large web corpora, such as Flickr, as described by Kollar et al. (2010b).

The $\mu_{k}$ are the weights corresponding to the output of a particular feature function. At training time, we observe SDCs, groundings $\Gamma$, and the output vector $\Phi$. In order to learn the parameters $\mu_{k}$ that maximize the likelihood of the training data set, we use L-BFGS (Andrew and Gao 2007) to optimize the parameters of the model through gradient descent.

Figure 5 shows an entire worked example for the command "Put the pallet on the truck," beginning with SDCs, the grounding graph, and finally the factorization of the distribution. Note that the factor graph contains subgraphs corresponding to the constituents "on the truck" (shown in figure 4) and "the truck" (shown in figure 3). This decomposition allows the model to learn word meanings from each factor and flexibly compose them together in order to understand novel commands.

\section{Results}

We present results from experiments with the $G^{3}$ framework using three corpora of natural language commands paired with robot actions and environment state sequences. Examples from the corpora appear in figure 6 . We used one part of these corpora to train the $\mathrm{G}^{3}$ model to learn the meanings of words and used a held-out test set to evaluate the end-to-end performance of the system at composing word meanings in order to follow commands.

The first corpus focuses on spatial prepositions describing paths, such as "across," "to," "toward," and "along." Each example in the corpus consists of a trajectory, a landmark object, and a phrase such as "Go to the door" or "Go across the confer-

$$
\begin{aligned}
\operatorname{PLACE}_{2}(r & =\text { on } \\
l_{1} & \left.=O B J_{1}(f=\text { the truck })\right)
\end{aligned}
$$

a. SDC Tree

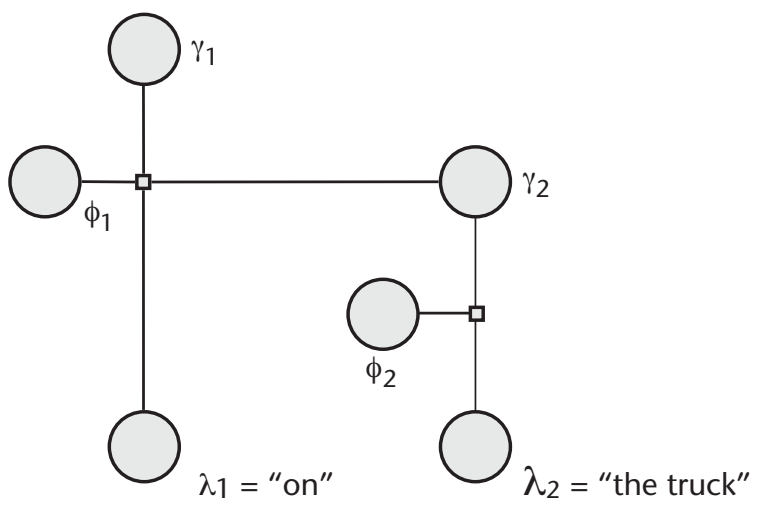

b. Induced Model

$p(\Phi \mid \Lambda, \Gamma, m)=p\left(\phi_{1} \mid \lambda_{1}, \gamma_{1}, \gamma_{2}, m\right) \times p\left(\phi_{2} \mid \lambda_{2}, \gamma_{2}, m\right)$

c. Factorization

Figure 4. SDCs and Grounding Graph for the Phrase "on the Truck."

ence room"; the corpus includes both positive and negative examples of each spatial relation. One of the authors created the corpus by drawing a sequence of waypoints that corresponded to a phrase such as "down the hallway." Negative examples were created by treating positive examples of one spatial relation as negative examples of another, with some exceptions such as "to" and "toward." This data set provides a simple test bed to demonstrate the model's performance, as well as providing training examples for bootstrapping the model on this important class of words. Figure $6 \mathrm{a}$ shows a sample prepositional phrase from this corpus, paired with a path and landmark.

The second corpus consists of natural language route instructions. We collected a corpus of 150 natural language route instructions from 15 people, through one floor of two adjoining office buildings. An example set of directions from the corpus is shown in figure $6 \mathrm{~b}$. Following these directions is challenging because they consist of natural language constrained only by the task and as a result may use any of the complicated linguistic structures associated with free-form natural lan- 


$$
\begin{aligned}
\operatorname{EVENT}_{1}(r & =\text { Put, } \\
I & =O B I_{2}(f=\text { the pallet }), \\
I_{2} & =\operatorname{PLACE}_{3}(r=\text { on, } \\
I & \left.\left.=O B J_{4}(f=\text { the truck })\right)\right)
\end{aligned}
$$

\section{a. SDC Tree}

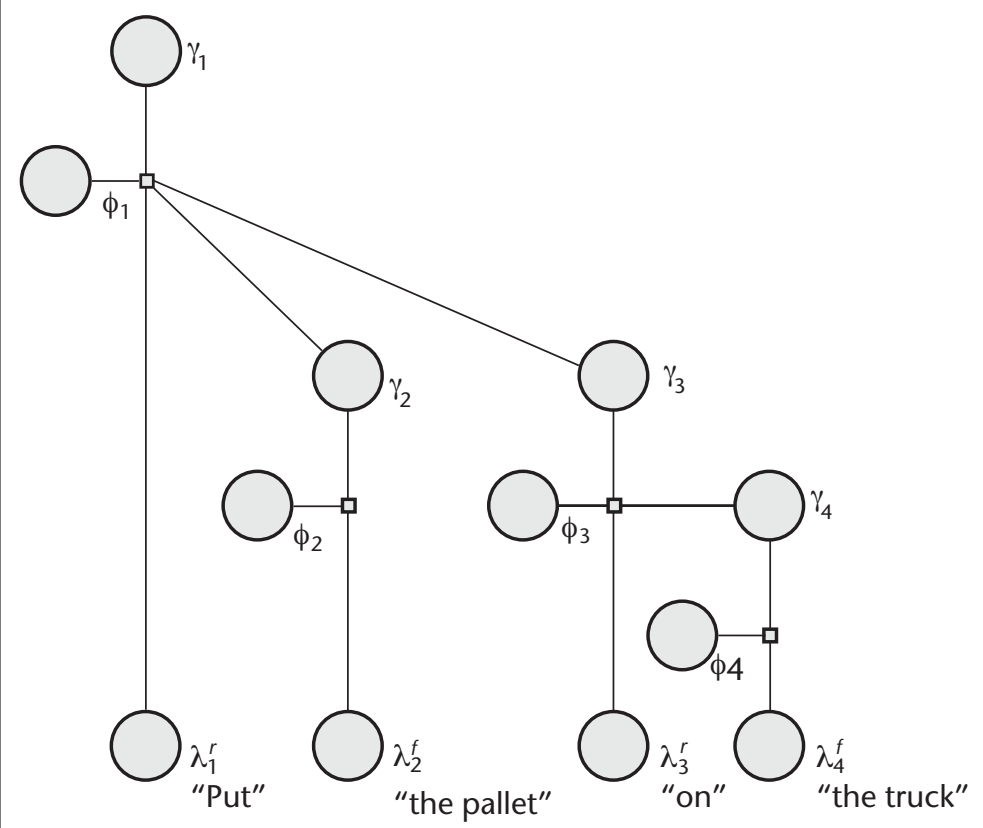

\section{b. Induced Model}

$p(\Phi \mid \Gamma$, SDCs, $m)=p\left(\phi_{1} \mid \gamma_{1}, \gamma_{2}, \gamma_{3}, \lambda_{1}^{r}=\right.$ Put,$\left.m\right) \times$

$$
\begin{aligned}
& p\left(\phi_{2} \mid \gamma_{2}, \lambda_{2}^{f}=\text { the pallet, } m\right) \times p\left(\phi_{3} \mid \gamma_{3}, \gamma_{4}, \lambda_{3}^{r}=\text { on, } m\right) \times \\
& p\left(\phi_{4} \mid \gamma_{4}, \lambda_{4}^{f}=\text { the truck, } m\right)
\end{aligned}
$$

\section{c. Factorization}

Figure 5. Worked Example for the Command "Put the Pallet on the Truck."

guage. This corpus provides a complex sample of spatial language for a real-world task. To train the model, we annotated each constituent in the corpus with a corresponding path segment or landmark. We constructed negative examples by randomizing these annotations. Figure $6 \mathrm{~b}$ shows a sample command from the corpus.
The third corpus consists of mobile-manipulation commands given to a real robotic forklift. Annotators on Amazon Mechanical Turk watched a video of a simulated forklift performing an action, then wrote natural language commands they would give to an expert human operator in order to command the operator to carry out the actions in the video. This corpus consists of a rich variety of mobile-manipulation commands such as "Pick up the pallet of tires directly in front of the forklift." Figure 6c shows an example command from this dataset.

\section{Meanings for Words}

Next, we trained models for each of the corpora and evaluated their performance for specific words in a held-out test set, using the same features for all models and annotated parses. Table $1 \mathrm{a}$ shows the performance on words from the spatial relations corpus. Not surprisingly, it learned good models for the meanings of words in this simple corpus. To illustrate the learned models for individual words, we present the probability distribution as a heat map, where red is high probability and blue is low probability. Figure 7 shows maps for "to the truck," "past the truck" and "toward the truck," demonstrating that the system has learned nuanced models for these different words.

Table $1 \mathrm{~b}$ shows the performance of the trained system on individual examples from the route directions corpus. Performance is lower because this corpus contained fewer examples of individual spatial relations and was noisier in general. The effects of this noise can be seen in the heat map shown in figure $9 \mathrm{~b}$.

Finally, table 1c shows the performance of the system when trained on the mobile manipulation corpus. The system was able to learn good models for verbs such as "put" and "take" as well as spatial relations such as "to," "toward," and "on" from relatively few training examples.

The word "take" appeared in both the mobile manipulation corpus and the route directions corpus, but it was used in different ways. In the route directions corpus, it was used in phrases such as "Take your first left," while in the mobile manipulation corpus, it was used in commands like "Take the pallet of tires to the trailer on the left." Although the system learned these two senses separately, learning from a single corpus that contained both would be challenging because the same feature weights would be trained for both word senses simultaneously.

Figure 8 a shows the distribution of locations for "on" as learned from the mobile manipulation corpus from phrases such as "Put the pallet on the truck." (The target locations are a constant height above the ground.) The system gives high weight to locations that are supported by the truck, 
because features related to "support" have the highest weight among the learned features for "on." Figure 8b shows the distributions for the phrase "near the truck," which is not peaked as strongly as "on." The distributions are asymmetric with respect to the truck because of frame-of-reference features that take into account the position and orientation of the robot. We intended these features to capture phrases like "on your left" and "to the left of," but the system also weights them for "on" and "near."

Figure 9 shows maps for "to the truck" from models trained on each of the three datasets. The system is able to learn good models from both the spatial relation and mobile manipulation data sets. The mobile manipulation data set is noisier because it contains fewer training examples, and many of the examples were part of compound prepositional phrases such as "to the left of the truck." The route instructions corpus is biased to go past the landmark object, probably because examples of "to" often occurred in the context of longer phrases such as "walk to the end of the hall and turn left."

\section{End-to-End Evaluation}

The fact that the model performed well at predicting the correspondence variable from annotated SDCs and groundings is promising but does not necessarily translate to good end-to-end performance when using the model to follow natural language commands.

To assess end-to-end performance, we evaluated the system in the mobile manipulation domain as described by Tellex et al. (2011). For each command in the corpus, the system inferred a plan and executed it in a realistic robot simulator. Then, annotators ranked whether the robot's behavior was correct or incorrect given the command. By this metric, our system correctly followed 54 percent of the 30 most confident commands in the corpus. When using a ground-truth parse instead of an automatic parse, the system followed 47 percent of commands from the entire corpus, and 63 percent of the 30 most confident commands.

The system qualitatively produced compelling end-to-end performance. When the system did make mistakes, it was often partially correct. For example, it might pick up the left tire pallet instead of the right one. Other problems stemmed from ambiguous or unusual language in the corpus commands, such as "remove the goods" or "then swing to the right," that make the inference particularly challenging. Despite these limitations, however, the system successfully followed commands such as "put the tire pallet on the truck," "pick up the tire pallet," "put down the tire pallet," and "go to the truck," using only data from the corpus to learn the model.

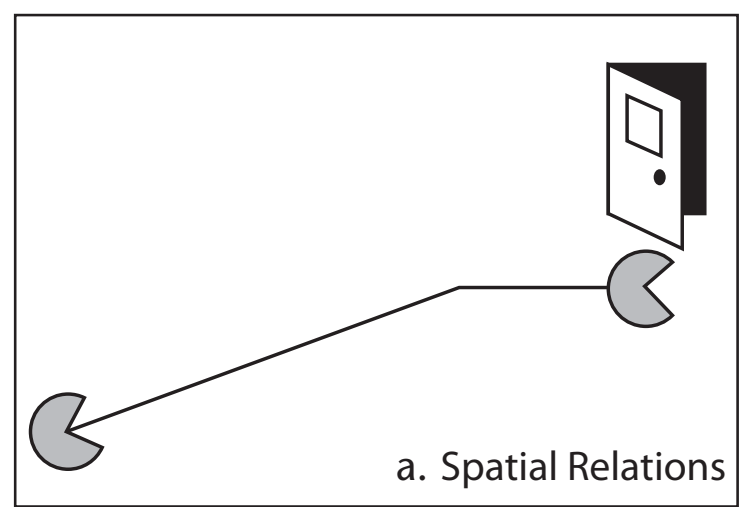

Go to the door.

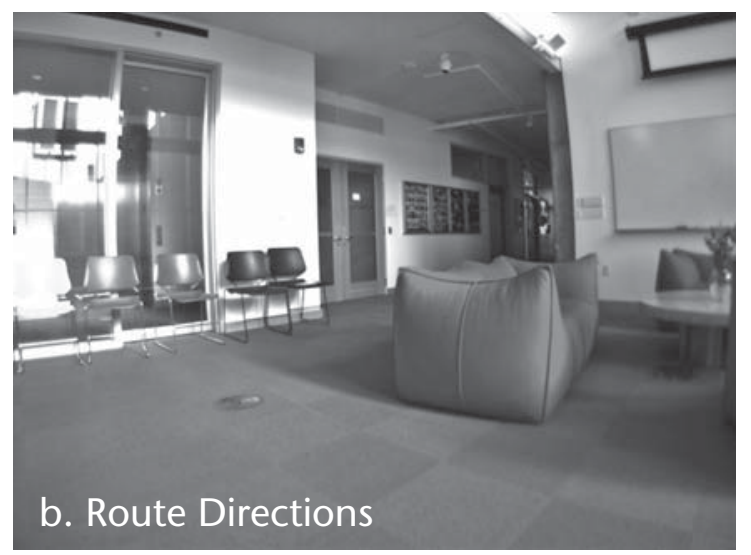

With your back to the windows, walk straight through the door near the elevators. Continue to walk straight, going through one door until you come to an intersection just past a white board.

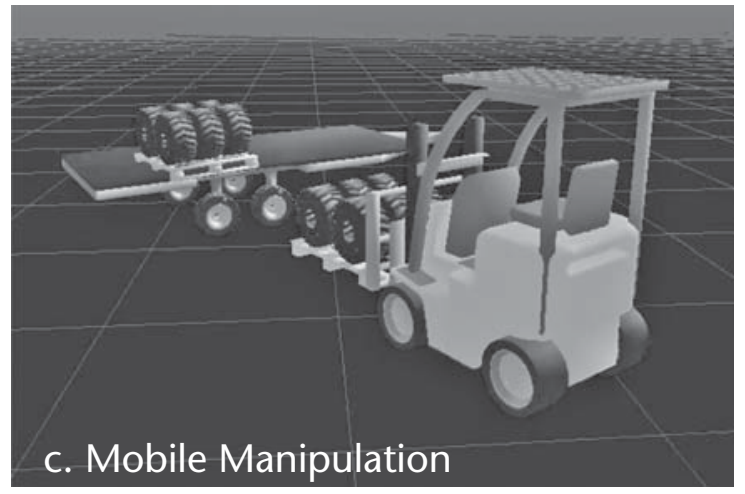

Lift the tire pallet in the air, then proceed to deposit it to the right of the tire pallet already on the table right in front of you.

Figure 6. Commands Paired with Environments from Corpora Used in Our Experiments. 


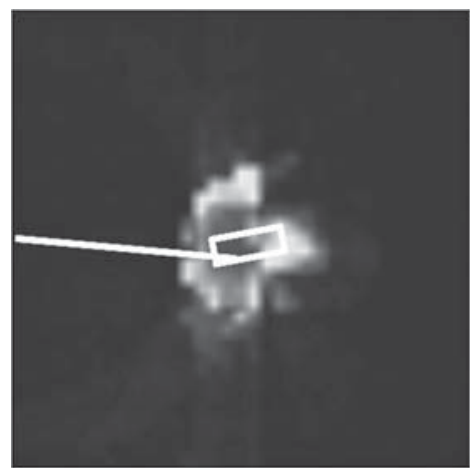

a. "to the truck"

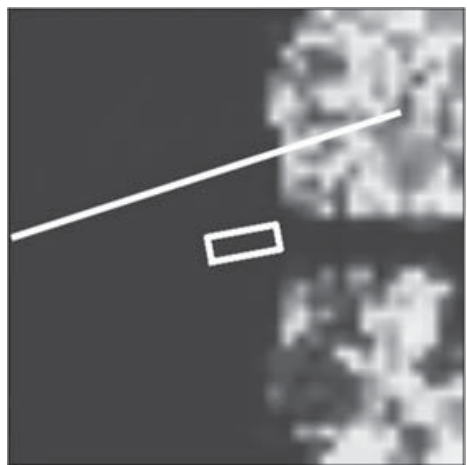

b. "past the truck"

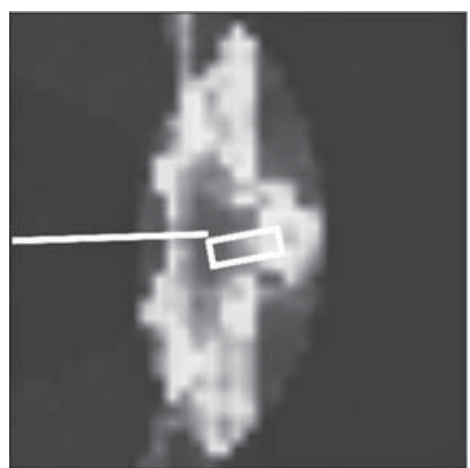

c. "toward the truck"

Figure 7. Heat Maps Showing High-Probability and Low-Probability Ending Locations for Various Phrases According to a Model Trained on the Spatial Relations Data Set.

The path is constrained to be a straight line starting at the left edge of the image. The highest-probability path is drawn in white.

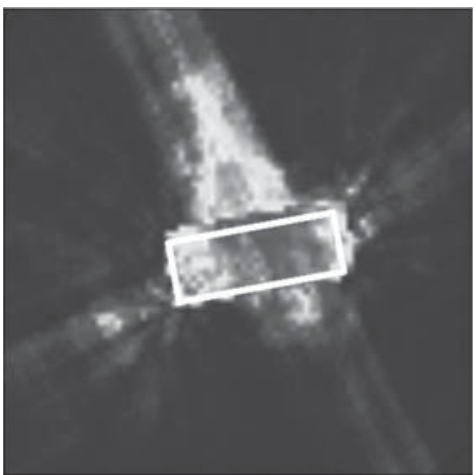

a. "on the truck"

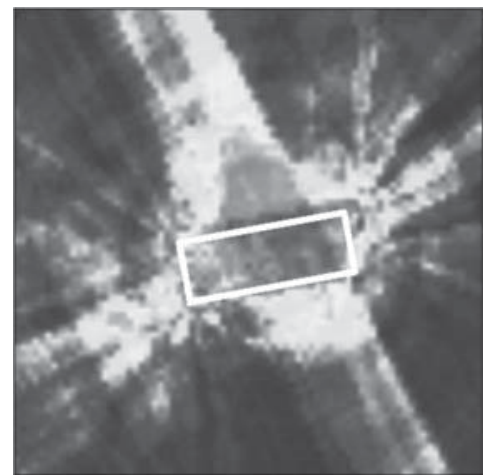

b. "near the truck"

Figure 8. Heat Map Showing High-Probability and Low-Probability Locations According to a Model Trained on the Mobile Manipulation Corpus.

The location of the truck is drawn in white.

An enabling technology for our approach to mobile manipulation is the availability of infrastructure for reliably simulating and logging robot actions. We used these technologies to collect corpora of language paired with robot actions to train the system. We were then able to simulate the robot and automatically produce videos of the system following each command in the corpus, which we used for the end-to-end evaluation.

\section{Lessons Learned}

An important next step is to leverage larger corpora of language paired with robot actions. Children hear millions of words in many different contexts as they acquire language. The relative lack of data was the cause of many of the errors our system made. For example, an annotator referred to a pallet that was separated from other pallets as "the 


\begin{tabular}{|c|c|c|c|c|}
\hline \multicolumn{5}{|c|}{ a. Spatial Relations } \\
\hline & Word & F-Score & Accuracy & No. of Examples \\
\hline \multicolumn{5}{|l|}{ path prepositions: } \\
\hline & across & 0.77 & 0.83 & 42 \\
\hline & around & 1.00 & 1.00 & 218 \\
\hline & past & 0.71 & 0.98 & 218 \\
\hline & through & 0.75 & 0.83 & 24 \\
\hline & to & 0.93 & 0.99 & 474 \\
\hline & toward & 0.84 & 0.99 & 214 \\
\hline \multicolumn{5}{|c|}{ b. Route Directions } \\
\hline & Word & F-Score & Accuracy & No. of Examples \\
\hline \multicolumn{5}{|l|}{ path prepositions: } \\
\hline & across & 0.75 & 0.75 & 8 \\
\hline & around & 0.80 & 0.80 & 10 \\
\hline & past & 0.80 & 0.83 & 30 \\
\hline & through & 0.81 & 0.81 & 114 \\
\hline & to & 0.72 & 0.71 & 144 \\
\hline & toward & 0.61 & 0.69 & 29 \\
\hline \multicolumn{5}{|l|}{ place prepositions: } \\
\hline & near & 1.00 & 1.00 & 8 \\
\hline & on & 0.98 & 0.98 & 55 \\
\hline \multicolumn{5}{|l|}{ verbs: } \\
\hline & take & 0.92 & 0.93 & 40 \\
\hline \multicolumn{5}{|c|}{ c. Mobile Manipulation } \\
\hline & Word & F-Score & Accuracy & No. of Examples \\
\hline \multicolumn{5}{|l|}{ path prepositions: } \\
\hline & to & 0.78 & 0.79 & 48 \\
\hline & toward & 0.80 & 0.75 & 4 \\
\hline \multicolumn{5}{|l|}{ place prepositions: } \\
\hline & near & 0.00 & 0.50 & 4 \\
\hline & on & 0.66 & 0.66 & 62 \\
\hline verbs: & & & & \\
\hline & lift & 0.88 & 0.87 & 60 \\
\hline & put & 1.00 & 1.00 & 6 \\
\hline & take & 1.00 & 1.00 & 12 \\
\hline
\end{tabular}

Table 1. Performance of the Learned Model in Terms of Recognizing Actions for Various Words.

The final column shows the number of examples in the test set, with a 70 percent-30 percent training-testing split.

lonely pallet," but the word "lonely" did not appear in the training set. As a result, the system was unable to learn a model for this word. Our learning framework requires detailed alignment annotation between linguistic constituents and groundings in the world, which limits our ability to leverage larger data sets. Our next goal is to reduce the amount of annotation required by using algorithms that alternate between picking labels and learning models using the inferred labels. A second approach to 


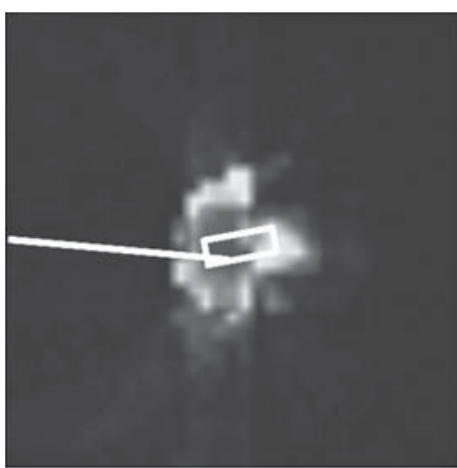

a. Spatial relations

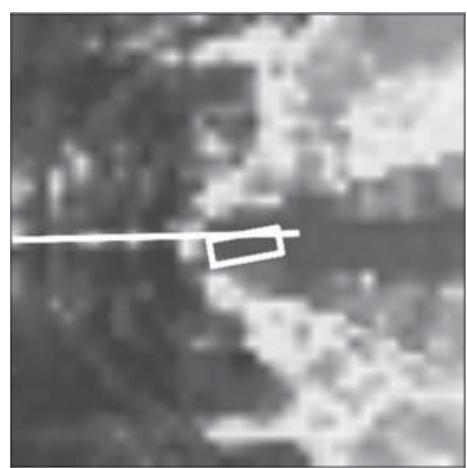

b. Route directions

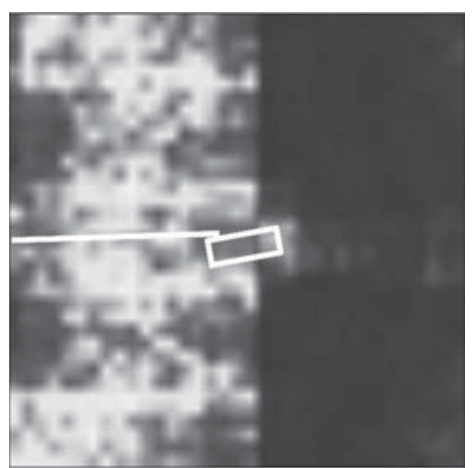

c. Mobile manipulation

Figure 9. Heat Maps Showing High- and Low-Probability

Ending Locations for a Path Corresponding to "To the Truck."

The path is constrained to be a straight line starting at the left edge of the image.

The highest probability path is drawn in white.

this problem would be to acquire word meanings from existing large corpora (Kollar and Roy 2009). The challenge here is to identify data sets that would allow the system to map from words such as "pick up" or "lonely" to actions and perceptual features accessible to the robot.

A second challenge is interpreting high-level commands such as "unload the truck" that might require long sequences of primitive actions, as well as lowlevel commands such as "drive forward six inches." An action space detailed enough to represent actions such as driving forward a small distance will require extremely long action sequences to generate behavior like unloading a truck. This problem was made concrete by one of our annotators, who posted instructions for picking up a dime with a forklift:

Raise the forks 12 inches. Line up either fork in front of the dime. Tilt the forks forward 15 degrees. Pull the truck forward until one fork is directly over the dime. Completely lower the forks. Put the truck in reverse and gently travel backward a foot. The dime will flip up backwards onto the fork. Level the forks back to 90 degrees. Raise the dime with the forks 12 inches.

To handle different granularities of actions, we are developing a hierarchical action space and new search algorithms that will enable the robot to efficiently search among both large-scale and smallscale actions when following a command.

A third challenge is learning word meanings that generalize across different domains without retraining the model. Figure 9 shows three different meanings for the word "to" learned from three different data sets. A further challenge is modifying learned models in response to modifiers, such as "half-way to the truck." Modeling nuanced changes of meaning in different contexts remains a challenging problem.

The ability to understand spatial language discourse and engage in dialogue is critical to enable robots to robustly interact with humans using language. The model described here represents an early step toward a framework for acquiring word meanings, but much remains to be done. A system that can understand the full complexity of language must be able to handle ellipsis (when words are omitted from sentences), conditional expressions (for example, "if a truck comes in, unload it"), and quantifiers (for example, "move all the tire pallets"). It must also reason about uncertainty from the speech recognizer about what the person actually said, as well as uncertainty in the parser, such as ambiguous prepositional phrase attachment. We envision a joint search over speech-recognition candidates, parse structures, and groundings in the world, applying information from multiple modalities jointly to reduce uncertainty. Furthermore the system must be able to combine multiple utterances into higher-level semantic units. Finally, it must be embedded in a higher-level dialogue understanding framework that can reason about the system's uncertainty and take actions to reduce it, such as asking questions. Grounding graphs provide a building block to address these problems, but a more sophisticated framework must be developed to utilize them effectively. 


\section{Conclusion}

This article describes two probabilistic approaches to the symbol-grounding problem. We first reviewed a generative model that factors according to the sequential structure of language. Next we presented a hierarchical model, called generalized grounding graphs $\left(\mathrm{G}^{3}\right)$, that is able to learn word meanings from corpora and compose them to understand novel commands. We described applications of the $\mathrm{G}^{3}$ framework to several different domains and presented results demonstrating that it has learned the meanings of complex spatial prepositions and verbs.

\section{Acknowledgments}

We would like to thank Dimitar Simeonov, Alejandro Perez, and Nick dePalma, as well as the annotators on Amazon Mechanical Turk and the members of the Turker Nation forum. This work was sponsored by the Robotics Consortium of the U.S. Army Research Laboratory under the Collaborative Technology Alliance Program, Cooperative Agreement W911NF-10-2-0016, and by the Office of Naval Research under MURI N00014-07-1-0749.

\section{Note}

1. www.flickr.com

\section{References}

Andrew, G., and Gao, J. 2007. Scalable Training of L1-Regularized Log-Linear Models. In Proceedings of the TwentyFourth International Conference on Machine Learning $(I C M L)$. New York: Association for Computing Machinery.

Bailey, D. 1997. When Push Comes to Shove: A Computational Model of the Role of Motor Control in the Acquisition of Action Verbs. Ph.D. Dissertation, Department of Computer Science, University of California, Berkeley, CA.

Branavan, S. R. K.; Chen, H.; Zettlemoyer, L. S.; and Barzilay, R. 2009. Reinforcement Learning for Mapping Instructions to Actions. In Proceedings of the 49th Annual Meeting of the Association for Computational Linguistics, 8290. Stroudsberg, PA: Association for Computational Linguistics.

Branavan, S.; Silver, D.; and Barzilay, R. 2011. Learning to Win by Reading Manuals in a Monte-Carlo Framework. In Proceedings of the 50th Annual Meeting of the Association for Computational Linguistics. Stroudsberg, PA: Association for Computational Linguistics.

Bugmann, G.; Klein, E.; Lauria, S.; and Kyriacou, T. 2004. Corpus-Based Robotics: A Route Instruction Example. Proceedings of the 8th Intelligent Autonomous Systems Conference, 96-103. Amsterdam: IOS Press.

de Marneffe, M.; MacCartney, B.; and Manning, C. 2006. Generating Typed Dependency Parses from Phrase Structure Parses. In Proceedings of the International Conference on Language Resources and Evaluation (LREC), 449-454. Paris: European Language Resources Association.

Dzifcak, J.; Scheutz, M.; Baral, C.; and Schermerhorn, P. 2009. What to Do and How to Do It: Translating Natural
Language Directives into Temporal and Dynamic Logic Representation for Goal Management and Action Execution. In Proceedings of the 2009 IEEE International Conference on Robotics and Automation (ICRA), 4163-4168. Piscataway, NJ: The Institute of Electrical and Electronics Engineers.

Ge, R., and Mooney, R. J. 2005. A Statistical Semantic Parser That Integrates Syntax and Semantics. In Proceedings of the Ninth Conference on Computational Natural Language Learning, 9-16. Stroudsburg, PA: Association for Computational Linguistics.

Harnad, S. 1990. The Symbol Grounding Problem. Physica D 42(1-3): 335-346.

Hsiao, K.-Y.; Mavridis, N.; and Roy, D. 2003. Coupling Perception and Simulation: Steps Towards Conversational Robotics. In Proceedings of the 2003 IEEE/RSJ International Conference on Intelligent Robots and Systems, volume 1, 928-933. Piscataway, NJ: The Institute of Electrical and Electronics Engineers.

Jackendoff, R. S. 1983. Semantics and Cognition, 161-187. Cambridge, MA: The MIT Press.

Kollar, T., and Roy, N. 2009. Utilizing Object-Object and Object-Scene Context When Planning to Find Things. In Proceedings of the 2009 IEEE International Conference on Robotics and Automation (ICRA), 4116-4121. Piscataway, NJ: The Institute of Electrical and Electronics Engineers. Kollar, T.; Tellex, S.; Roy, D.; and Roy, N. 2010a. Grounding Verbs of Motion in Natural Language Commands to Robots. In Proceedings of the 12th International Symposium on Experimental Robotics (ISER). Berlin: Springer.

Kollar, T.; Tellex, S.; Roy, D.; and Roy, N. 2010b. Toward Understanding Natural Language Directions. In Proceedings of the 5th ACM/IEEE International Conference on Human-Robot Interaction (HRI), 259-266. New York: Association for Computing Machinery.

Kollar, T.; Tellex, S.; and Roy, N. 2010. A Discriminative Model for Understanding Natural Language Route Directions. In Dialog with Robots: Papers from the AAAI Fall Symposium. Technical Report FS-10-05. Menlo Park, CA: AAAI Press.

Kress-Gazit, H., and Fainekos, G. E. 2008. Translating Structured English to Robot Controllers. Advanced Robotics 22(12): 1343-1359.

Kschischang, F. R.; Frey, B. J.; and Loeliger, H.-A. 2001. Factor Graphs and the Sum-Product Algorithm. IEEE Transactions on Information Theory 47(2): 498 -519.

Lafferty, J. D.; McCallum, A.; and Pereira, F. C. N. 2001. Conditional Random Fields: Probabilistic Models for Segmenting and Labeling Sequence Data. In Proceedings of the International Conference on Machine Learning (ICML), 282-289. San Francisco: Morgan Kaufmann Publishers.

MacMahon, M.; Stankiewicz, B.; and Kuipers, B. 2006. Walk the Talk: Connecting Language, Knowledge, and Action in Route Instructions. In Proceedings of the Twenty-First National Conference on Artificial Intelligence (AAAI), 1475-1482. Menlo Park, CA: AAAI Press.

Marocco, D.; Cangelosi, A.; Fischer, K.; and Belpaeme, T. 2010. Grounding Action Words in the Sensorimotor Interaction with the World: Experiments with a Simulated iCub Humanoid Robot. Frontiers in Neurorobotics 4(7).

Matuszek, C.; Fox, D.; and Koscher, K. 2010. Following Directions Using Statistical Machine Translation. In Pro- 
ceedings of the 5th ACM/IEEE International Conference on Human-Robot Interaction (HRI), 251-258. New York: Association for Computing Machinery.

Modayil, J., and Kuipers, B. 2007. Autonomous Development of a Grounded Object Ontology by a Learning Robot. In Proceedings of the Twenty-Second AAAI Conference on Artificial Intelligence, 1095-1101. Menlo Park, CA: AAAI Press.

Regier, T. P. 1992. The Acquisition of Lexical Semantics for Spatial Terms: A Connectionist Model of Perceptual Categorization. Ph.D. Dissertation, Department of Computer Science, University of California at Berkeley.

Roy, D. 2005. Semiotic Schemas: A Framework for Grounding Language in Action and Perception. Artificial Intelligence 167(1-2): 170-205.

Roy, D.; Hsiao, K.; and Mavridis, N. 2003. Conversational Robots: Building Blocks for Grounding Word Meanings. Proceedings of the HLT-NAACLO3 Workshop on Learning Word Meaning from Non-Linguistic Data. Stroudsberg, PA: Association for Computational Linguistics.

Roy, D.; Patel, R.; DeCamp, P.; Kubat, R.; Fleischman, M.; Roy, B.; Mavridis, N.; Tellex, S.; Salata, A.; Guinness, J.; Levit, M.; and Gorniak, P. 2006. The Human Speechome Project. In Proceedings of the 28th Annual Cognitive Science Conference, 192-196. Wheat Ridge, CO: Cognitive Science Society, Inc.

Shimizu, N., and Haas, A. 2009. Learning to Follow Navigational Route Instructions. In Proceedings of the 21st International Joint Conference on Artifical Intelligence, 14881493. Menlo Park, CA: AAAI Press.

Sugita, Y., and Tani, J. 2005. Learning Semantic Combinatoriality from the Interaction Between Linguistic and Behavioral Processes. Adaptive Behavior - Animals, Animats, Software Agents, Robots, Adaptive Systems 13(1): 3352.

Tellex, S.; Kollar, T.; Dickerson, S.; Walter, M.; Banerjee, A.; Teller, S.; and Roy, N. 2011. Understanding Natural Language Commands for Robotic Navigation and Mobile Manipulation. In Proceedings of the Twenty-Fifth AAAI Conference on Artificial Intelligence. Menlo Park, CA: AAAI Press.

Tellex, S.; Kollar, T.; Shaw, G.; Roy, N.; and Roy, D. 2010. Grounding Spatial Language for Video Search. In International Conference on Multimodal Interfaces and the Workshop on Machine Learning for Multimodal Interaction, ICMIMLMI '10, 31:1-31:8. New York: Association for Computing Machinery.

Vogel, A., and Jurafsky, D. 2010. Learning to Follow Navigational Directions. In Proceedings of the 48th Annual Meeting of the Association for Computational Linguistics (ACL), 806-814. Stroudsburg, PA: Association for Computational Linguistics.

Winograd, T. 1970. Procedures as a Representation for Data in a Computer Program for Understanding Natural Language. Ph.D. Dissertation, Massachusetts Institute of Technology, Boston, MA.

Stefanie Tellex is a research scientist at the MIT Computer Science and Artificial Intelligence Laboratory at MIT. As part of her Ph.D. dissertation at the MIT Media Lab, Tellex developed models for the meanings of spatial prepositions and motion verbs. She has presented her work at SIGIR, HRI, AAAI, and ICMI, describing systems for searching surveillance video with spatial language queries and for giving instructions to mobile robots.

Thomas Kollar has a Ph.D. in electrical engineering and computer science (EECS) from the Massachusetts Institute of Technology (MIT). His thesis concerned learning to understand spatial natural language commands and his research interests include robot learning, language grounding, and human-robot interaction. He was the general chair of the HRI Pioneers Workshop at the 6th ACM/IEEE International Conference on Human-Robot Interaction.

Steven Dickerson graduated from MIT in 2011 with undergraduate degrees in computer science and aerospace engineering. He is currently employed by Goldman Sachs.

Matthew R. Walter is a research scientist in the Computer Science and Artificial Intelligence Laboratory at MIT. He received his Ph.D. in mechanical engineering from the Massachusetts Institute of Technology and the Woods Hole Oceanographic Institution in 2008. His research interests include perception, motion planning, and human-robot interaction, so as to enable mobile robots to operate safely and effectively within unstructured environments.

Ashis Gopal Banerjee is a postdoctoral associate in the Computer Science and Artificial Intelligence Laboratory at MIT. He completed his Ph.D. in mechanical engineering at the University of Maryland in 2009. He received the 2009 Best Dissertation Award from the Department of Mechanical Engineering and the 2009 George Harhalakis Outstanding Systems Engineering Graduate Student Award at the University of Maryland. His research interests include planning under uncertainty, machine learning, and micro and nano manipulation.

Seth Teller is a professor in the Department of Electrical Engineering and Computer Science, and a member of the Computer Science and Artificial Intelligence Laboratory, at MIT. He received his Ph.D. in computer science from the University of California, Berkeley in 1992. Teller's research interests include machine perception, mobile manipulation, human-robot interaction, and assistive technology.

Nicholas Roy is an associate professor in the Department of Aeronautics and Astronautics at the Massachusetts Institute of Technology and a member of the Computer Science and Artificial Intelligence Laboratory at MIT. He received his Ph.D. in robotics from Carnegie Mellon University in 2003. His research interests include mobile robotics, decision making under uncertainty, humancomputer interaction, and machine learning. 